\title{
Subphotospheric Structure of Sunspots and Active Regions
}

\author{
Alexander G. Kosovichev \\ Stanford University, Stanford, CA 94305, USA email: AKosovichev@solar.stanford.edu
}

\begin{abstract}
New methods of local area helioseismology provide three-dimensional maps of soundspeed variations and mass flows in the upper convection zone, giving important insight into the internal structure and dynamics of sunspots and active regions. Most of these results are obtained from SOHO/MDI data using the method of time-distance helioseismology (or acoustic tomography). Robustness of this method has been significantly improved by incorporating most important wave propagation effects and stochastic properties of solar oscillations. Time-distance helioseismology reveals that developed sunspots have a two layer structure: a relatively thin subphotospheric layer of lower sound speed, and a deeper layer of higher sound speed. The mass flows in the upper layer are typically converging and directed downward, while in the deeper interior the flows are mostly diverging. These results support the cluster model of sunspots suggested by Parker. New observations also provide interesting information about emerging magnetic flux, formation and evolution of active regions and complexes of activity, and allow us to investigate effects of sub-photospheric dynamics of active regions on the global circulation of the Sun and also small-scale rapid shear flows associated with flares and CME.
\end{abstract}

\section{Method}

Just below the photosphere, turbulent convection excites acoustic waves which propagate deep into the solar interior. Because the sound speed increases with depth these waves are reflected and come back to the solar surface. Time-distance helioseismology measures travel times of acoustic waves propagating to different distances, and uses these measurements to infer variations of the wave speed along the wave paths. The wave speed depends on temperature, magnetic field strength and flow velocity field in the region of the wave propagation. By measuring reciprocal travel times of acoustic waves propagating along the same ray paths in the opposite directions, and then taking the mean and the difference of these travel times it is possible to separate the flow velocity (advection) effect from temperature and magnetic field perturbations (Kosovichev \& Duvall, 1997). However, in order to disentangle contributions of temperature and magnetic field to the mean travel times it is necessary to measure the travel-time anisotropy, and this has not been accomplished. Therefore, the current helioseismic results represent maps of sub-photospheric variations of the sound (magneto-acoustic) speed and flow velocities. In addition to acoustic waves turbulent convection excites surface gravity waves which propagate in a shallow sub-surface layer, and allow us to measure the wave speed perturbations and horizontal flow field in this layer (Gizon 2003).

The travel times are typically measured from a cross-covariance function of solar oscillation signals for various distances and time lags. When for a given distance the time lag corresponds to the propagation time of acoustic waves for this distance, a wavepacketlike signal appears in the cross-covariance function. The cross-covariance plotted as a function of the distance and time lag displays a set of ridges formed by the wave-packet signals (Fig. 1a). Since the solar oscillations are stochastic it is necessary to use the oscillation signals at least 4-8 hour long and also average them over some surface (typically, 


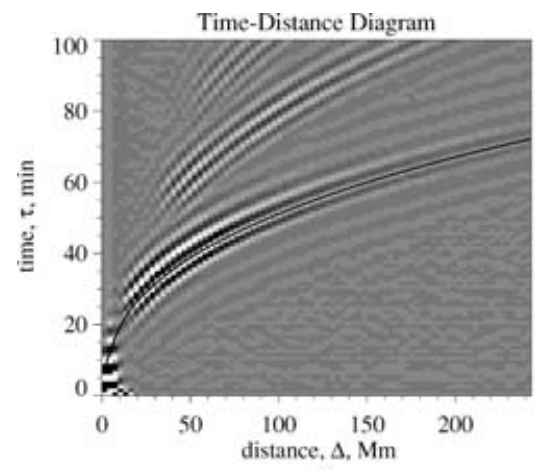

a)

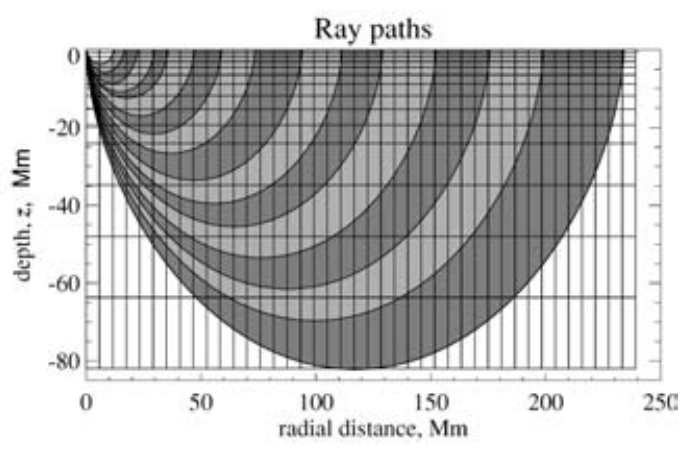

b)

Figure 1. a) Cross-covariance functions of solar oscillations as a function of distance between measurement points on the solar surface and time lag. The lowest ridge is formed by the acoustic waves packets propagating between these points through the solar interior. The solid curve shows the time-distance relation in the ray approximations. The higher ridges are formed by the wave packets with additional bounces at the surface. b) a sample of acoustic ray paths used for time-distance helioseismology, shown in a vertical plane. The shadowed regions illustrate ranges of averaging. The vertical and horizontal lines show a grid used for inversion of acoustic travel time data.

circular) areas in order to obtain a sufficient signal-to-noise ratio. Then, the travel times are determined by fitting a wavelet to this function (e.g. Kosovichev \& Duvall, 1997), or by measuring displacement of the ridges (Gizon \& Birch, 2002).

The relationship between the observed travel-time variations and the internal properties of the Sun is given by so-called sensitivity kernels through integral equations. These integral equations are solved by standard mathematical inversion techniques such as LSQR and Multi-Channel Deconvolution (MCD) (Couvidat et al. 2004). The sensitivity functions are calculated using a ray theory (Fig. 1b) or more complicated wave perturbation theories, e.g. Born approximation, which takes into account the finite wave-length effects (Birch \& Kosovichev, 2000). These theories can also take into account stochastic properties of acoustic sources distributed over the solar surface (Gizon \& Birch, 2002; Birch et al. 2004.)

\section{Results}

\subsection{Sub-surface structure of sunspots}

The high-resolution data from SOHO/MDI have allowed us to investigate structures and flows beneath sunspots in detail (Kosovichev et al. 2000; Zhao et al. 2001). Figure 2 shows an example of the internal structure of a large sunspot observed on June 20, 1998. An image of the sunspot taken in the continuum is shown at the top. The sound-speed perturbations under the sunspot are quite strong, reaching $3 \mathrm{~km} / \mathrm{s}$. Beneath the spot the perturbation is negative in the subsurface layers, but it becomes positive in the deeper interior. The negative perturbations of the acoustic wave speed beneath the spot are, probably, due to the lower temperature. It follows that magnetic inhibition of convection that makes sunspots cooler is most effective within the top 2-3 $\mathrm{Mm}$ of the convection zone. The strong positive perturbation below suggests that the deep sunspot structure is hotter than the surrounding plasma by $\sim 10^{3} \mathrm{~K}$. These data also show, at a depth of $4 \mathrm{Mm}$, connections to the spot of small pores, A and B, which have the same magnetic 

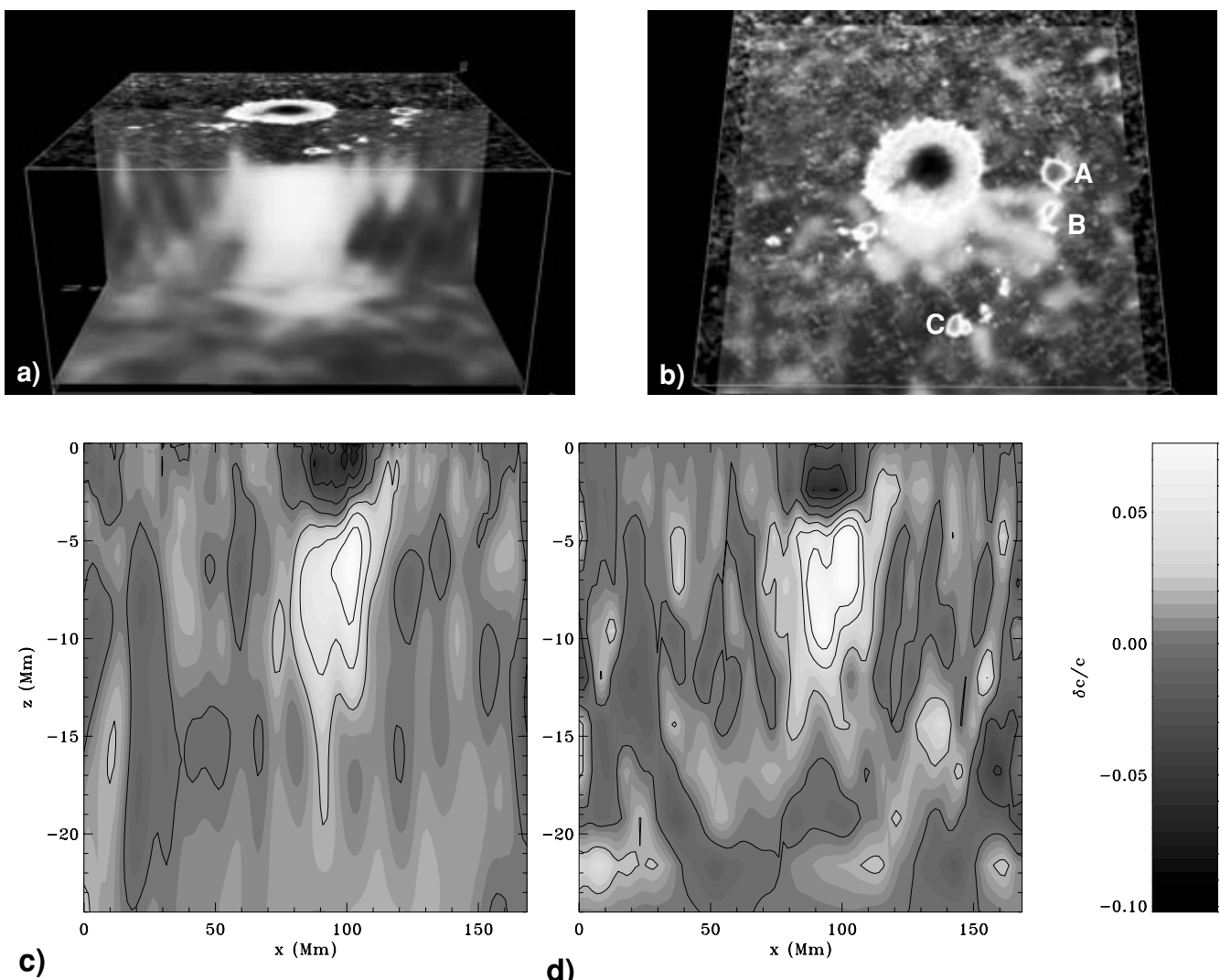

d)

Figure 2. The sound-speed perturbation in a large sunspot observed on June 20, 1998, are shown as vertical and horizontal cuts. The horizontal size of the box is 13 degrees $(158 \mathrm{Mm})$, the depth is $24 \mathrm{Mm}$. The positive variations of the sound speed are shown in white and light gray, and the negative variations (just beneath the sunspot) in dark. The semitransparent top in panels a)and b) is the surface intensity image (dark color shows umbra, and light color shows penumbra). In panel b)the horizontal sound-speed plane is located at the depth of $4 \mathrm{Mm}$, and shows long narrow structures ('fingers') connecting the main sunspot structure with surrounding pores A and B of the same magnetic polarity as the spot. Pores of the opposite polarity (e.g. C) are not connected to the sunspot. (Kosovichev et al. 2000). The lower panels show a cut in the vertical $(\mathrm{x} ; \mathrm{z})$ plane of the inversion results for the relative variation of the sound speed, obtained with different sensitivity kernels: c) Fresnel-zone kernels and d) ray-approximation kernels. Contour lines emphasize the structure of the sunspot. (Couvidat et al. 2004).

polarity as the main spot. Pore $\mathrm{C}$ of the opposite polarity is not connected. This suggests that sunspots represent a tree-like structure in the upper convection zone.

\subsection{Sunspot flow patterns}

Figure 3 shows flow maps beneath the sunspot. In Fig.3a which shows results for the subphotospheric layer $3 \mathrm{Mm}$ deep, one can clearly identify a ring of strong downflows around the sunspot, with relatively weaker downflows inside the ring. Converging flows at the sunspot center can also be seen. Fig.3b shows the flows in the layer, corresponding to a depth of 6-9 Mm. The sunspot region is occupied by a ring of upflows with almost zero velocity at the center. At the same time, strong outflows from the sunspot center can be seen. Clearly, the motion to the South-East direction is much stronger.

Figures $3 \mathrm{c}$-d show two vertical cuts. Converging and downward flows can be seen in both diagrams right below the sunspot region from $1.5 \mathrm{Mm}$ to about $5 \mathrm{Mm}$. Below 


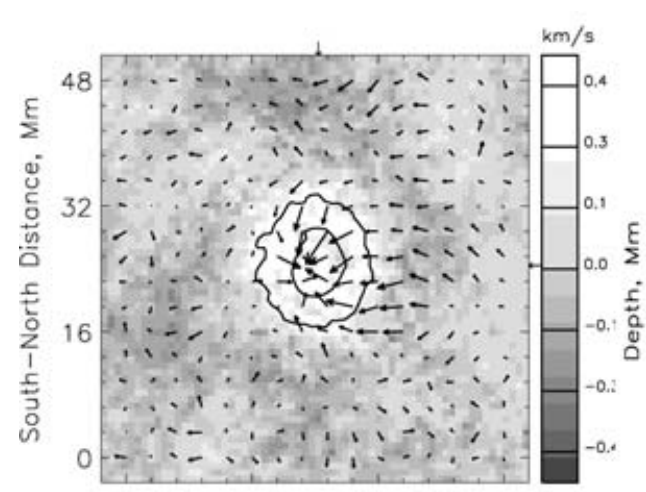

a)

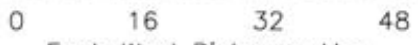

Eost-West Distance, Mm

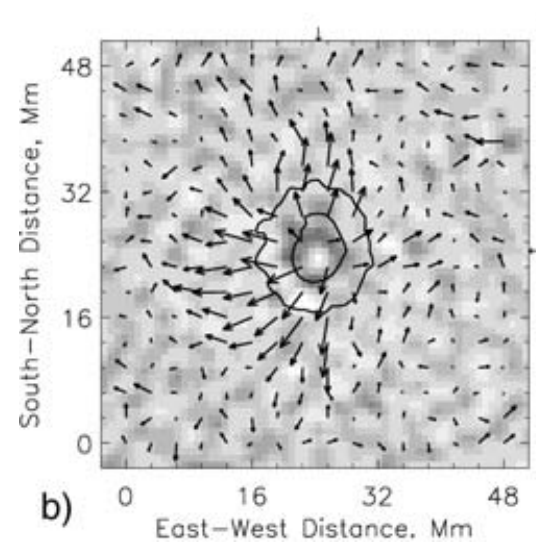

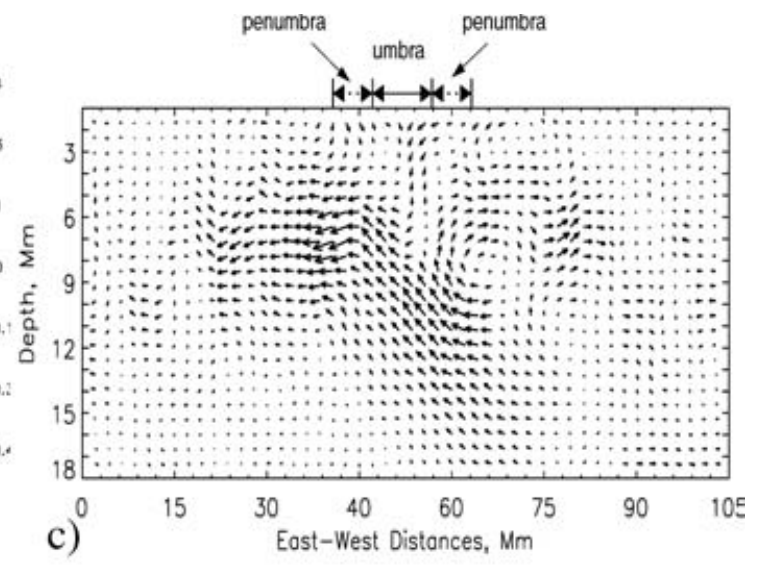

$\mathrm{km} / \mathrm{s}$

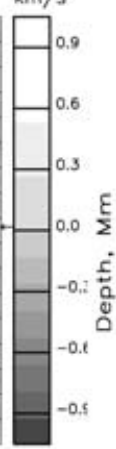

d)

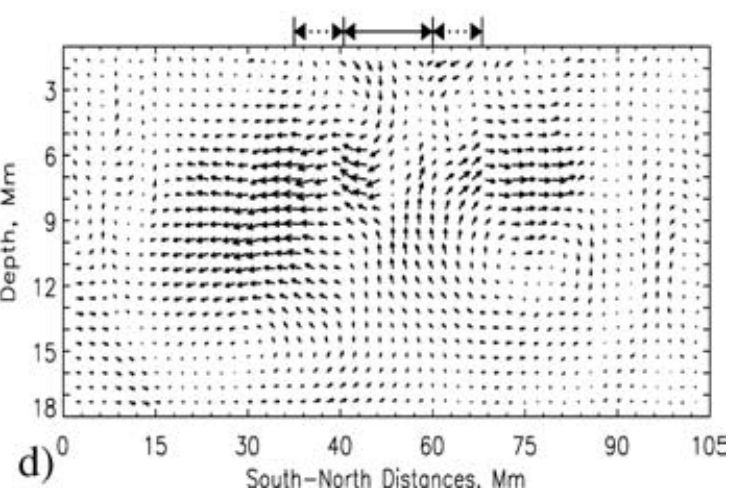

Figure 3. Plasma flows below a sunspot observed on on Dec 6, 1998, at the depth of 0-3 Mm (a) and depth of 6-9 Mm (b). The arrows show the magnitude and directions of the horizontal flows, and the background gray-scale map shows the vertical flows: white color shows downflows, and dark color shows upflows. The contour lines at the center show the boundaries of umbra and penumbra. The longest arrow corresponds to $1.0 \mathrm{~km} / \mathrm{s}$ in panel a), and $1.6 \mathrm{~km} / \mathrm{s}$ in panel b). The small arrows outside the frame indicate where the vertical cuts shown in panels c) and d) are made. The vertical cuts are made through the sunspot center in the East-West (c) South-North (d) directions. (Zhao et al. 2001)

that, the horizontal outflows seem to dominate in this region, though relatively weaker upflows also appear. These inversion results show converging flows in the top layer of the sunspot region which are opposite to the well-known diverging Evershed flow observed spectroscopically on the solar surface. Gizon (2003) has studied the horizontal nearsurface flows in sunspots using the f-mode diagnostics (Fig.4). The velocity amplitude of the Evershed flow inferred from the f-mode is systematically lower than that at the surface. This suggests that the Evershed flow is a shallow phenomenon. This can explain the apparent disagreement with the results obtained from acoustic modes.

The observational evidence of converging flows beneath the sunspot supports the cluster model of sunspots suggested by Parker (1979). According to this model, magnetic field of a sunspot consists of many separate tubes below the surface, and that a converging downward flow beneath the sunspot holds the separate tubes in a loose cluster (Fig.5c). The helioseismology results are also consistent with the MHD model of Hurlburt \& Rucklidge (2000) illustrated in Fig.5b and 5d. 


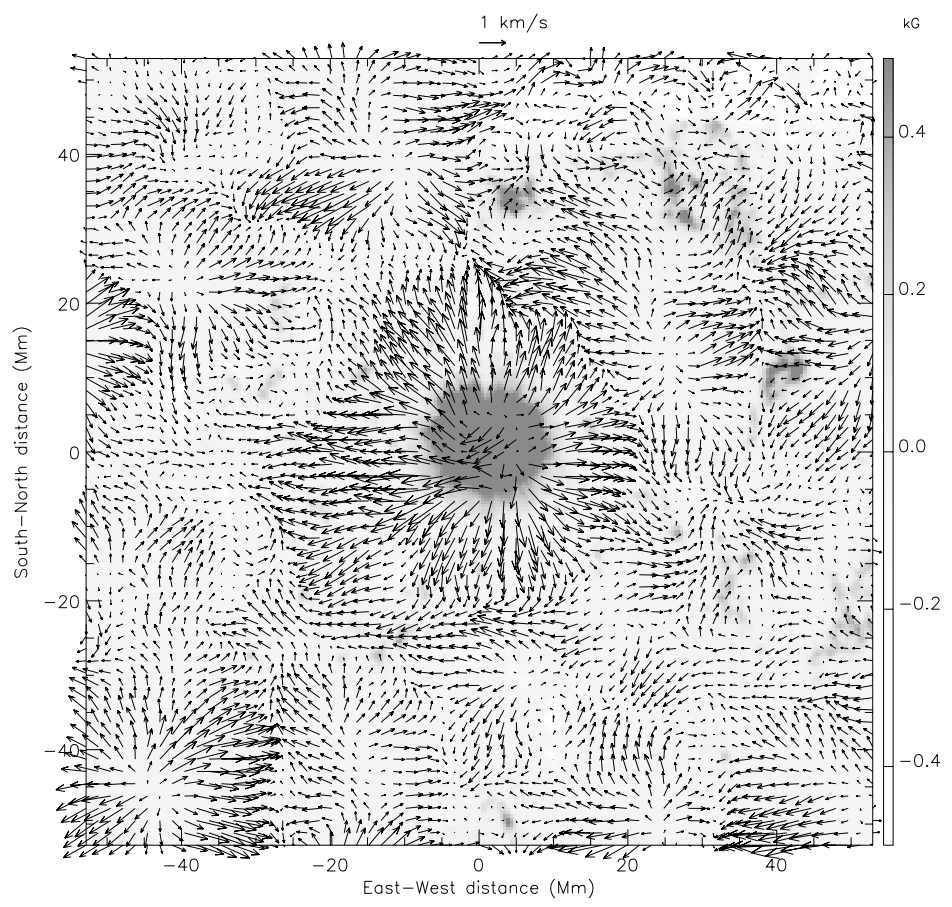

Figure 4. Horizontal near-surface flows around a sunspot of Dec 6, 1998, obtained with f-mode time-distance helioseismology (Gizon 2003). The background map is the line-of-sight magnetogram. The outflow corresponding to the surface moat flow is clearly visible.

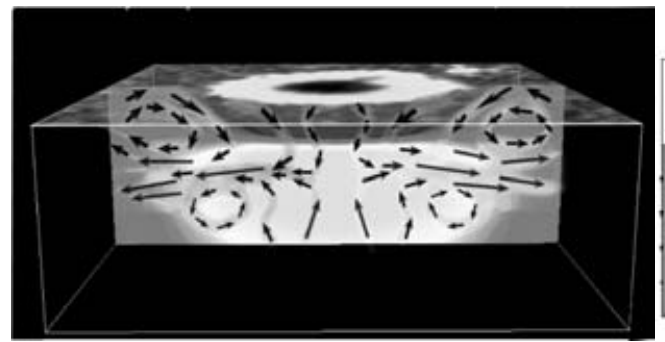

a)

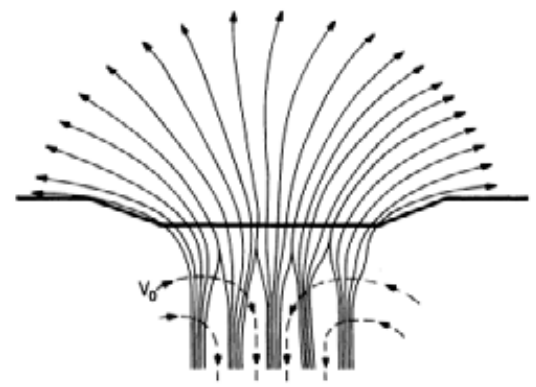

c)

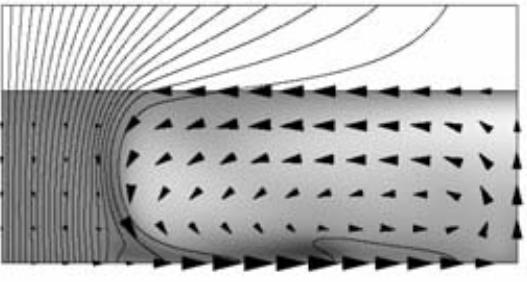

b)

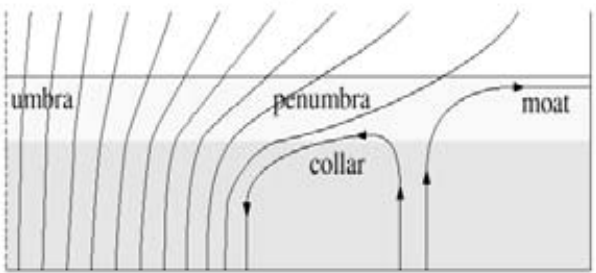

d)

Figure 5. a) Schematic picture showing the axisymmetrical component of subphotospheric sunspot flows. The background vertical image shows the sound-speed variation (in this case, the light color corresponds to positive perturbation, and the dark color shows negative perturbations beneath the surface). The top panel shows a white-light image of this sunspot. b) Mass flow in an axisymmetrical MHD model of Hurlburt \& Rucklidge (2000). c) the cluster model of sunspots (Parker, 1979). d) a schematic picture of sunspot flows, consistent with the helioseismology results and the MHD model (Hurlburt \& Rucklidge, 2000). 

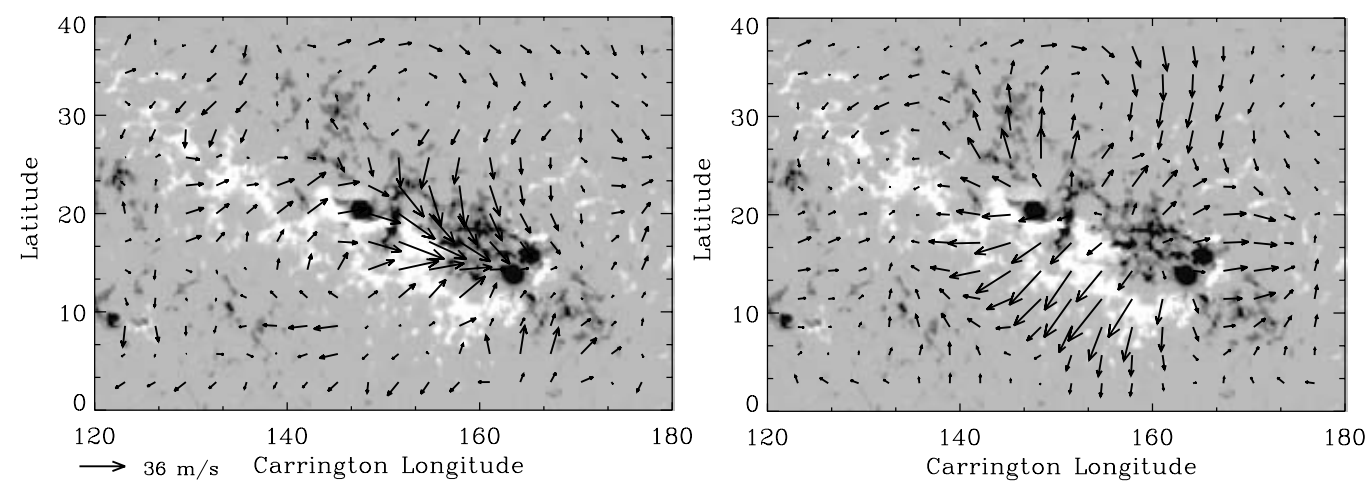

Figure 6. The large-scale averaged flow maps for a large active region AR9433 at two different depth intervals: $0-3 \mathrm{Mm}$ (left) and 9-12 Mm (right). (Zhao \& Kosovichev, 2004).

\subsection{Dynamics of active regions}

Converging flow patterns are also observed on larger scale around active regions (Fig. 6) by both time-distance helioseismology (Zhao \& Kosovichev, 2004) and by the ringdiagram method (Haber et al, 2002). These large-scale flows affect the global dynamics of the Sun, and magnetic flux transport. In particular, these flows result in significant changes in the mean meridional flow with the solar cycle.

Helioseismic observations also show that the flow dynamics changes during the evolution of active regions. Figure 7 shows the horizontal and vertical components of plasma flows $2 \mathrm{Mm}$ below the photosphere during emergence, maximum activity phase and decay of NOAA 9393 which was the largest active region of solar cycle 23. During its emergence these observations do not reveal a large-scale diverging upflow which could be associated with a large magnetic structure coming up from the deep interior. Instead, a converging flow around the emerging active region is quickly formed. During the maximum of activity these observations show large-scale converging downflows accompanied by complicated shearing motions which be related to flaring activity (Kulinova et al. 2003). During the decay phase the downflows become significantly weaker, and diverging flows around decaying sunspots are observed.

Figure 8 illustrates the sound-speed structure of large active region NOAA 10488. It reveals a loop-like structure of the active region below the surface.

\section{Conclusions and perspectives}

The initial results of local helioseismology have revealed complicated structures and flow patterns associated with sunspots and active regions in the upper convection zone. They show formation of large-scale vortex flows converging around large sunspots and active regions in the near-surface layers and diverging in the deeper interior. The characteristic speed of these flows in sunspots may reach $2 \mathrm{~km} / \mathrm{s}$, and on larger scale of active regions the flow speed is about $50 \mathrm{~m} / \mathrm{s}$. The converging downflows beneath sunspots support Parker's cluster model of sunspots. The large-scale vortices affect the pole-ward meridional flow resulting in significant changes with the solar cycle, which may affect the magnetic flux transport to the polar regions and, thus, the process of the magnetic polarity reversals. On smaller scales, helioseismology reveals deep shearing and twisting flows in active regions, which appear to be associated with flaring activity. 

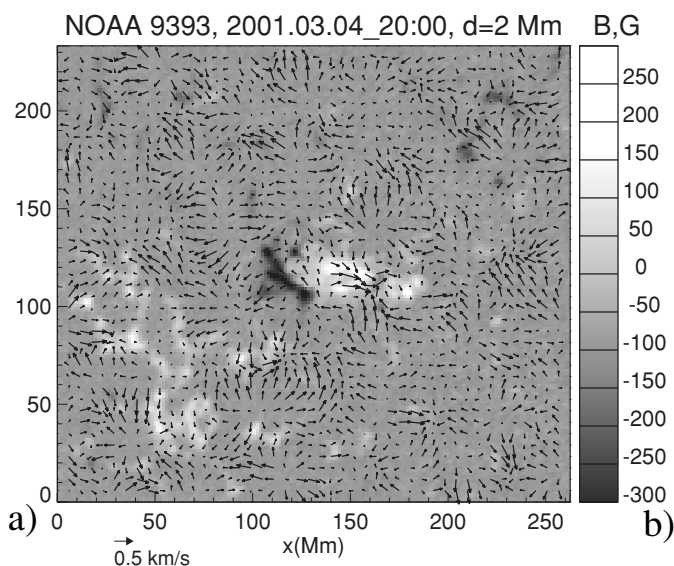

NOAA 9393, 2001.03.04_20:00, d=2 Mm Vz,km/s

a)
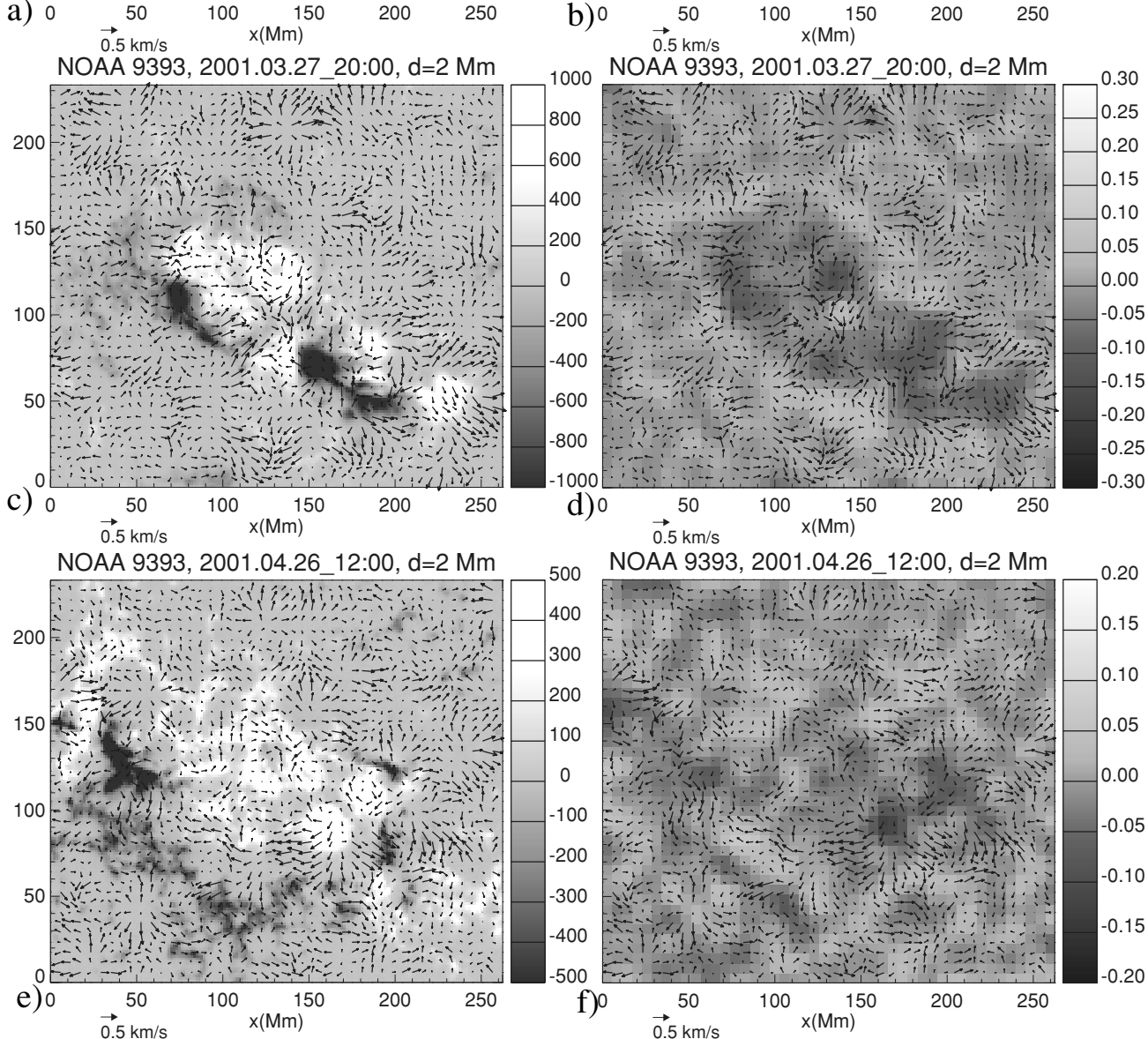

Figure 7. Evolution of active region NOAA 9393 (March-April 2001): a) and b)- emergence; c) and d) - the maximum activity, e) and f) - decay. Left panels show the photospheric magnetic field (grayscale map) and the horizontal velocity field at depth $2 \mathrm{Mm}$; the right panels show the vertical velocity in gray scale and the horizontal velocity field at the same depth.

It is important to confirm and refine these initial results by developing more precise data analysis and inversion procedures for the time-distance technique and other local helioseismology methods.

Helioseismic and Magnetic Imager (HMI) instrument on Solar Dynamics Observatory (SDO) will provide continuous high-resolution solar oscillation data which will allow us 

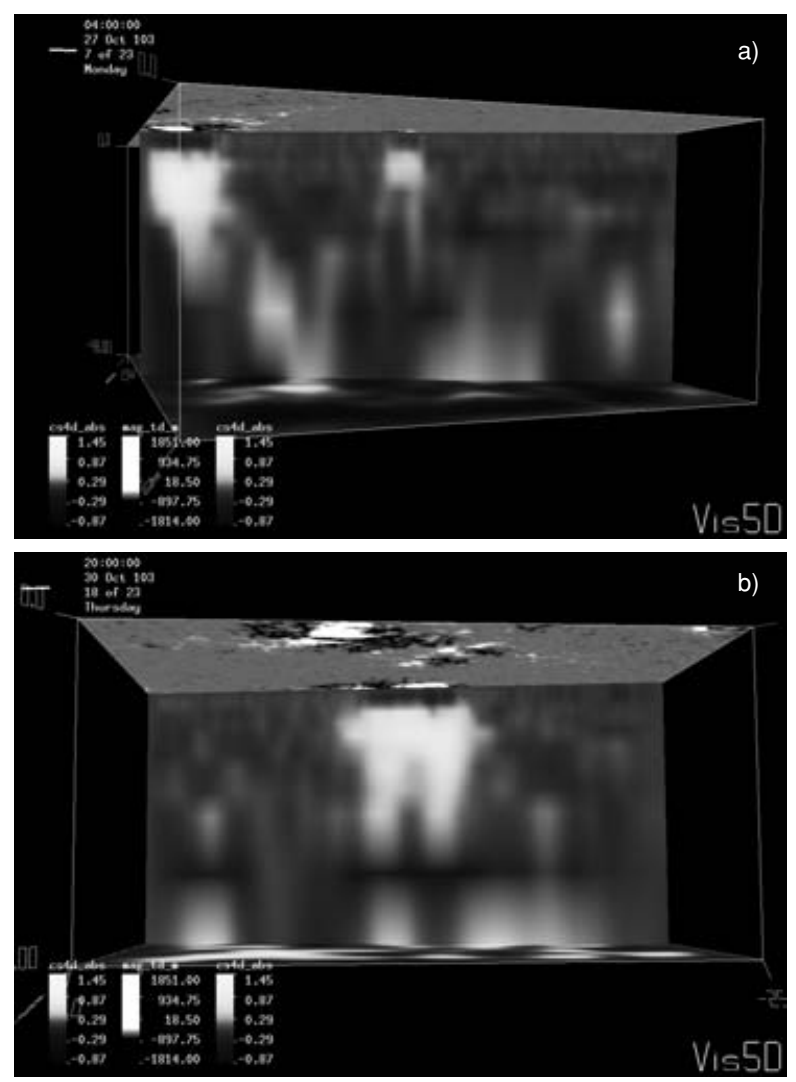

Figure 8. Sound-speed variations associated with developing active region NOAA 10488 (October 2003): a) emergence of the active region in the middle of the domain (the structure near the right boundary is AR10486; b) fully developed AR 10488. The depth of the box is $48 \mathrm{Mm}$, the horizontal size is about $540 \mathrm{Mm}$. The sound-speed scale is from -1 to $1.5 \mathrm{~km} / \mathrm{s}$, the scale of the photospheric field shown in the upper panel is from -1800 to 1800 Gauss.

to study the dynamics of subphotospheric structures and flows and determine their role in the formation, evolution and activity of solar magnetic structures at various scales.

\section{References}

Birch, A.C. \& Kosovichev, A.G. 2000 Solar Phys. 192, 193-201.

Birch, A.C., Kosovichev, A.G., \& Duvall, T.L., Jr. 2004 Astrophys. J. 608, 580-600.

Couvidat, S., Birch, A. C., Kosovichev, A. G., \& Zhao, J. 2004 Astrophys. J. 607, 554-563.

Gizon L. 2003 Probing flows in the upper solar convection zone Thesis (PhD) Stanford

Gizon, L. \& Birch, A.C. 2002 Astrophys. J. 571, 966-986.

Haber, D. A., Hindman, B. W., Toomre, J. \& Thompson 2002 Solar Phys. 220, 371-380.

Hurlburt, N.E. \& Rucklidge, A.M. 2000 Mon. Not. R. Astr. Soc. 314, 793-806.

Kosovichev, A.G., \& Duvall, T.L. Jr. 1997 In SCORe'96 : Solar Convection and Oscillations and their Relationship, (ed. F.P. Pijpers et al), Ast. Space Sci. Lib. vol.225, pp. 241-260.

Kosovichev, A. G., Duvall, T. L. \& Scherrer, P. H. 2000 Solar Phys. 192, 159-176.

Kulinova, A.,Dzifcakova,E., Duvall, T.L., Jr \& Kosovichev, A.G. 2003, In Solar variability as an input to the Earth's environment, (ed. A.Wilson), ESA SP-535, Noordwijk, 125 - 128.

Parker, E. N. 1979 Astrophys. J., 230, 905-923.

Zhao, J., Kosovichev, A.G. \& Duvall, T.L., Jr. 2001 Astrophys. J., 557, 384-388.

Zhao, J., \& Kosovichev, A. G. 2004 Astrophys. J., 603, 776-784. 